OPEN ACCESS

Edited by: Kailiang Zhou,

The Second Affiliated Hospital and Yuying Children's Hospital of Wenzhou Medical University, China

Reviewed by:

Peng Liangyu,

Nanjing Drum Tower Hospital, China M. Ahmed,

Phcog.Net, India

*Correspondence: Zhongyuan Xia xiazhongyuan2005@aliyun.com Jishi Ye

yee1989@whu.edu.cn

${ }^{+}$These authors have contributed equally to this work

Specialty section:

This article was submitted to Cell Death and Survival, a section of the journal Frontiers in Cell and Developmental

Biology

Received: 12 October 2021 Accepted: 15 November 2021

Published: 29 November 2021

Citation:

Chen J, Li X, Jia Y, Xia Z and Ye J (2021) Publication Trends on Mitophagy in the World and China: A

16-Year Bibliometric Analysis. Front. Cell Dev. Biol. 9:793772. doi: 10.3389/fcell.2021.793772

\section{Publication Trends on Mitophagy in the World and China: A 16-Year Bibliometric Analysis}

\author{
Jingli Chen ${ }^{1,2+}$, Xin $\mathrm{Li}^{3 \dagger}$, Yifan Jia ${ }^{1}$, Zhongyuan $\mathrm{Xia}^{1 *}$ and Jishi Ye ${ }^{1 *}$ \\ ${ }^{1}$ Department of Pain, Renmin Hospital of Wuhan University, Wuhan, China, ${ }^{2}$ Department of Anesthesiology, The Central Hospital \\ of Wuhan, Tongji Medical College, Huazhong University of Science and Technology, Wuhan, China, ${ }^{3}$ Department of \\ Anesthesiology, Hubei Cancer Hospital, Tongji Medical College, Huazhong University of Science and Technology, Wuhan, China
}

In the past 16 years, research on mitophagy has increasingly expanded to a wider range of subjects. Therefore, comprehensively analyzing the relevant progress and development trends on mitophagy research requires specific methods. To assess the hotspots, directions, and quality of results in this field worldwide, we used multiple tools to examine research progress and growing trends in research on the matter during the last 16 years (from 2005 to 2020). We also compared the quantity and quality of the literature records on mitophagy published by research institutions in China and other developed countries, reviewed China's contribution, and examined the gap between China and these developed countries. According to the results of our bibliometric analysis, the United States and its research institutes published the most papers. We identified cell biology as the most commonly researched subject on mitophagy and AUTOPHAGY as the most popular journal for research on mitophagy. We also listed the most cited documents from around the world and China. With gradually increased funding, China is progressively becoming prominent in the field of mitophagy; nevertheless, the gap between her and major countries in the world must be closed.

Keywords: mitophagy, bibliometric analysis, VOSviewer, web of science, gap

\section{INTRODUCTION}

Mitochondria, complex organelles in the cell, keep complex organisms alive and are important biochemical metabolism centers of cells (Zilocchi et al., 2019). A mitochondrion produces adenosine triphosphate through oxidative phosphorylation and provides a key intermediate for the biosynthesis of amino acids, hormones, and fatty acids. Cells develop complex and interconnected regulatory pathways to maintain mitochondrial homeostasis by balancing mitochondrial biogenesis and clearing out damaged mitochondria. Therefore, signaling pathways that regulate mitochondrial homeostasis are crucial to cell survival, and their dysfunction is closely associated with aging and the occurrence and development of major diseases, such as cancer, cardiovascular, and neurodegenerative diseases (Pamenter et al., 2018; Iwata, 2019).

Mitophagy is a form of autophagy that mediates the removal of defective or redundant mitochondria (Doxaki and Palikaras 2020; Wang et al., 2021). And it is the only known way to selectively remove entire mitochondria (D'Acunzo et al., 2019). Mitophagy can also inhibit the production of excessive reactive oxygen species and retain valuable nutrients (such as oxygen) so that they are not consumed inefficiently, thus promoting cell survival under various pathological conditions (Cai et al., 2012; Salimi et al., 2019; Ye et al., 2019). 
In the past decade, more and more investigations have been conducted on mitophagy. Therefore, comprehensively analyzing the relevant progress and development trend in the field of mitochondrial autophagy requires specific methods. Bibliometrics, an analysis technique based on the number of citations and citation models, can be used to evaluate the impact of various research findings, such as a journal article or the number of citations and research directions of a series of research results (Bernard et al., 2019; Chang et al., 2019; Garcovich and Adobes Martin, 2019). Before being employed in this manner, bibliometric analysis was widely used to assess progress and trends in different research fields, including anesthesiology (Ye et al., 2018), critical care medicine (Nakao et al., 2019), and urology (Zhao et al., 2019), and played an essential role in understanding current research hotspots and future trends.

Up to now, there have been no bibliometric reports on research trends and scientific outcomes in the field of mitophagy. VOSviewer is a software tool developed based on the Java programming language for building and visualizing bibliometric networks. These networks may include journal researchers or individual publications constructed using citation lists coupled to common citations or co-author relationships. VOSviewer also provides text mining capabilities for building and visualizing co-existing networks of important terms extracted from the scientific literature. This software can be used to build networks of scientific publications, scientific journals, and researchers who study and organize national keywords or terms. Moreover, VOSviewer provides three types of visual maps: network visualization, overlay visualization, and density visualization, which can enable the further understanding of the relationships between institutions, authors, and keywords (Lei et al., 2019).

To assess the hotspots, directions, and quality of mitophagyrelated results worldwide, we used bibliometrics to analyze research progress and development trends in this field during the last 16 years (from 2005 to 2020). We also compared the quantity and quality of the literature records in the field published by research institutions in China and other developed countries, reviewed China's contribution, and examined the gap between China and these developed countries.

\section{METHODS}

\section{Database}

This research was carried out in the same way as previous similar literature (Wang et al., 2019). All data were collected on January 28, 2021: data were downloaded from public databases. There were no ethical issues, with ethical approval not applicable for this study. Articles on mitophagy published from 2005 to 2020 were retrieved from PubMed and web of science (WOS) online databases. Impact factors (IF) were obtained from the citation report database of journals in 2019.

\section{Retrieval Strategy}

The search terms in WOS were as follows: TOPIC: (mitophagy), Refined by: DOCUMENT TYPES: (ARTICLE OR EDITORIAL
MATERIAL OR REVIEW OR PROCEEDINGS PAPER OR MEETING ABSTRACT OR LETTER) AND LANGUAGES: $($ ENGLISH), Databases $=$ WOS, BIOSIS, INSPEC, KJD, MEDLINE, RSCI, SCIELO Timespan = 2005-2020.

The search terms in PubMed were as follows: "mitophagy" [MeSH Terms] OR "mitophagy" [All Fields]) AND (bibliography [Filter] OR clinical study [Filter] OR journal article [Filter] OR letter [Filter] OR review [Filter]).

\section{Bibliometric Analysis}

Using the intrinsic function of WOS, we analyzed the trends in research publication and the quality of publications from 2005 to 2020. Factors scrutinized included country/region, institution distribution, and top 10 cited references with high citation frequency.

We also used VOSviewer (Van Eck\& Waltman, Leiden University, Netherlands) and Microsoft Excel to mine, map, and cluster data from the retrieved articles. The programs portrayed keywords and countries using various colors and sizes of circles based on the frequency of occurrence of a keyword or country in both the titles and abstracts.

\section{RESULTS}

\section{Trends in Publications on Mitophagy in the World and China}

From 2005 to 2020, a total of 5,829 and 5,338 papers on mitophagy were recorded in the WOS and PubMed databases, respectively, based on the above retrieval strategy. Because the included journals from the two databases are different in content, the results are unlikely to be similar. The number of related papers published each year shows a significant upsurge year after year, highlighting a gradually increasing interest in mitophagy over the past 16 years. Our inquiry revealed that researchers from 137 countries were involved in scientific studies on mitochondrial autophagy. United States-based investigators published the most papers $(2,225,38.171 \%)$ in the WOS database, followed by mainland China (1,503, 25.785\%), Japan (390, 6.691\%), England (391, 6.708\%), and Germany (362, 6.210\%). Among the top five countries with the largest amount of publications, the United States and mainland China experienced the most significant rise (Figures $\mathbf{1 A - C}$ ).

Using the VOSViewer software, we analyzed the cooperative relationships between all countries in the field of mitophagy. Per our findings, only 51 countries had published more than five papers at the time of our analysis, while there were 8 close relationships. Among them, the United States, mainland China, Japan, and South Korea cooperated closely. England, Portugal, Brazil, and Iran worked closely together too. Germany had frequent associations with Luxembourg, Sweden, Netherlands, and Ireland. Therefore, trans-national and trans-regional communication in the field of mitophagy is quite common. Combined with growth curves and collaboration networks, the United States remains at the center of the field, while China is playing an increasingly key role. 


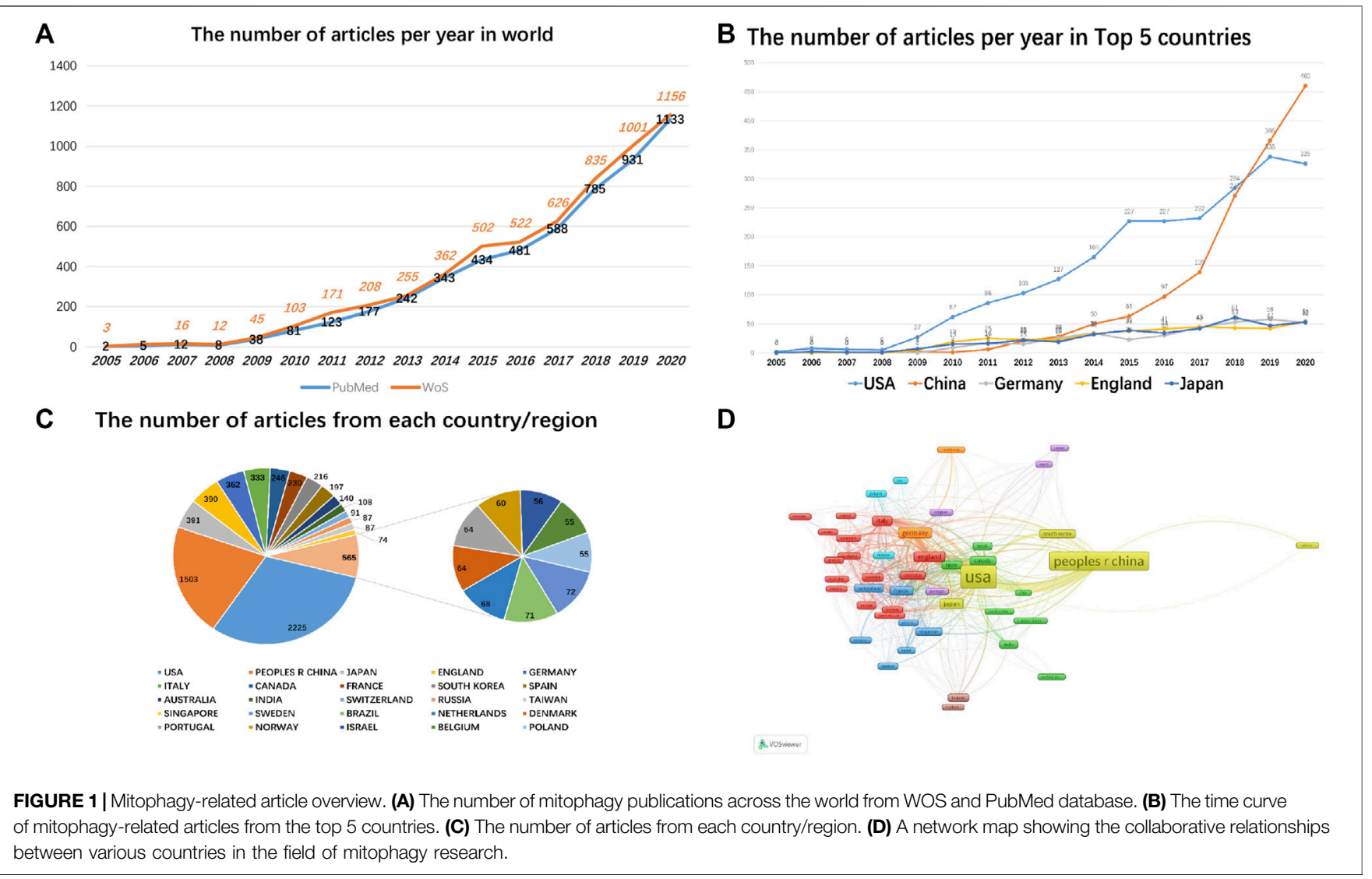

\section{Citation of Related Papers and H-Index Analysis}

From the perspective of institutions with relevant publications, the University of Pittsburgh, the University of California - San Diego, Chinese Academy of Sciences, University College London, and Fudan University have published the most papers on mitophagy in the world: the University of Pittsburgh leads the way in this category and had the highest $\mathrm{H}$-index and total citations, reflecting its prominent position in the field. Its paper "Loss of PINK1 Function Promotes Mitophagy through Effects on Oxidative Stress and Mitochondrial Fission", written by Dagda, Ruben K, and coauthors (Dagda et al., 2009) and published in 2009, was cited 615 times.

While the number of papers from Chinese organizations has increased rapidly, a gap remains between them and the world's top-tier countries and institutions. Among mainland Chinese establishments, the three institutions with the most published papers on mitophagy are the Chinese Academy of Sciences, Fudan University, and Zhejiang University. The Chinese Academy of Sciences has published up to 107 papers: its "Mitochondrial outermembrane protein FUNDC1 mediates hypoxia-induced mitophagy in mammalian cells", written by Liu, Lei, and coauthors (Liu et al., 2012) and published in 2012, has received 635 citations.

Using the VOSViewer software, we identified about 609 institutes with more than 5 published papers on mitophagy and about 137 institutes with more than 20 published papers. As shown in Figures 2E,F, our co-occurrence chart on research institutions revealed that the University of Pittsburgh has a close working relationship with some research establishments in China, including the Chinese Academy of Sciences, Central South University, and Zhejiang University, while Harvard University frequently collaborates with other American universities, like the University of California - San Diego. Per the coverage visualization chart, the most prominent research institutions on mitophagy have gradually shifted from European and American universities to research establishments in mainland China (Figures 2E,F).

\section{Research Direction in the Field of Mitophagy and Distribution of Journals}

Globally, AUTOPHAGY has published the most papers on mitophagy $(333,5.713 \%$ ) as of the time of our research, followed by FASEB JOURNAL $(130,2.23 \%)$ and SCIENTIFIC REPORTS (101, 1.733\%). According to the Q1 classification of Journal Citation Reports (2019 version), most of the top ten publishing journals were not among the very top journals in the world. China has a similar situation (Figures 3A,B).

In terms of research direction, investigations on mitophagy around the world focused on Cell Biology, Biochemistry, Molecular Biology, Neurosciences, Neurology, Science Technology, Other Topics, and Oncology. Comparable to the 


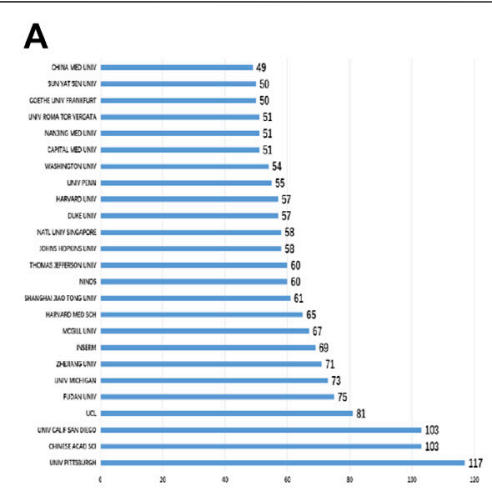

C

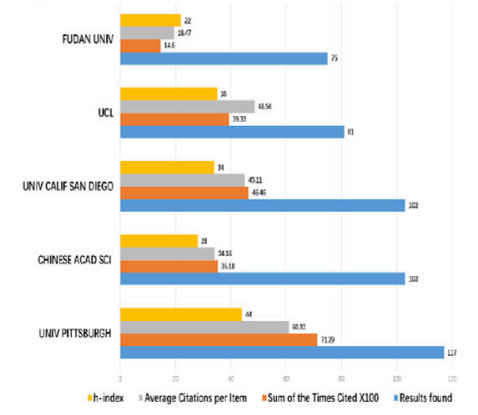

B

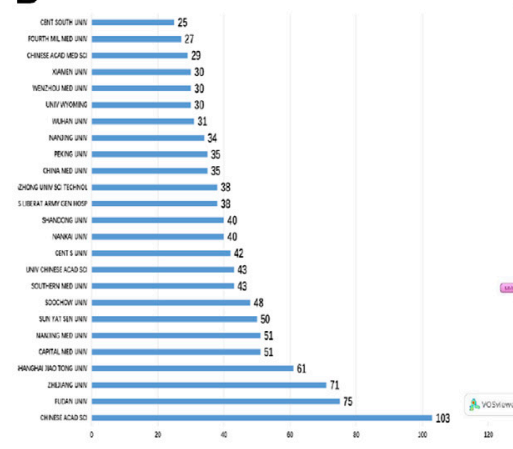

D

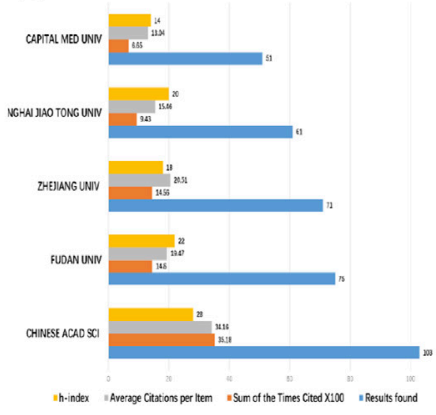

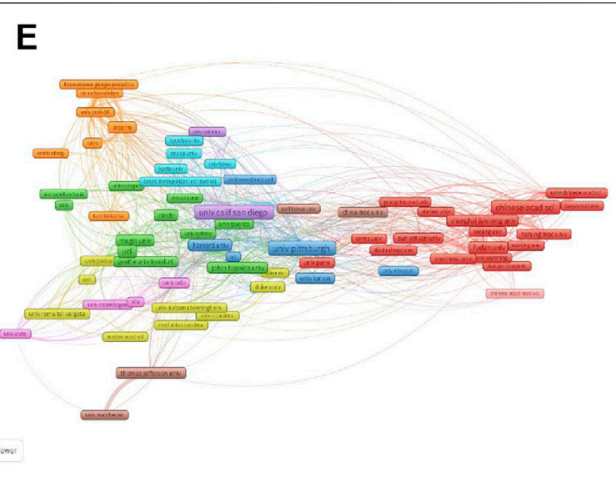

F

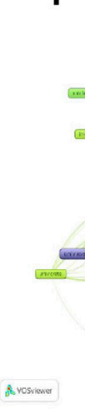

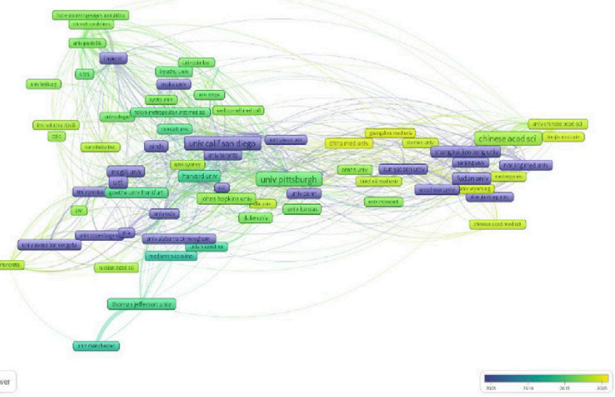

FIGURE 2 | Citations of related papers and H-index analysis. (A) The number of mitophagy publications from institutions across the world. (B) The number of mitophagy publications from Chinese institutions. (C) The total citations, average citations per paper, and $\mathrm{H}$-indexes for mitophagy articles from institutions across the world. (D) The total citations, average citations per paper, and $\mathrm{H}$-indexes for neuropathic pain articles from Chinese institutions. (E) A network map showing the collaborative relationships between various institutions in the field of mitophagy research. (F) A density map showing the collaborative relationships between various institutions in the field of mitophagy research.

research direction around the globe, research direction on mitophagy in mainland China also focuses on Cell Biology, Biochemistry, Molecular Biology, Oncology, Neurosciences, Neurology, and Pharmacology Pharmacy (Figures 3C,D).

\section{The Top 10 Most Cited Articles on Mitophagy in the World and China}

Using the built-in tools in the WOS database, we screened out the most cited papers with mitophagy-related subjects in the world and China, as shown in Table 1, 2. The two most cited articles abroad were reviews published in top journals. The paper "Pink1/ Parkin-mediated mitophagy is dependent on VDAC1 and P62/ SQSTM1", written by Geisler et al. (2010), was the most cited research paper in the past 15 years. Parkinson's disease, the most common neurodegenerative movement disorder, was the subject of discourse in this paper. Current research has identified PINK1 and Parkin mutations as the most common causes of Parkinson's disease. However, their molecular roles in the pathogenesis of the disease remain unclear. Per the investigation, the PINK1/Parkin pathway is a critical mechanistic step linking mitochondrial damage to ubiquitination and autophagy in non-neurons and neuronal cells. PINK1 kinase activity and its mitochondrial localization sequence are the prerequisites for inducing the translocation of E3 ligase Parkin to depolarized mitochondria. Notably, Geisler et al. identified voltage-dependent anion channel
1 (VDAC1) as a target for Parkin-mediated Lys 27 polyubiquitination and mitophagy. Overall, the paper established a functional association between Pink1-Parkin and mitochondrial selective autophagy, which is involved in the pathogenesis of Parkinson's disease.

China is a relatively late participant in mitophagy research, which explains the fewer number of cited papers published. Liu Lei et al.'s research paper "Mitochondrial outer-membrane protein FUNDC1 mediates hypoxia-induced mitophagy in mammalian cells", published in NATURE CELL BIOLOGY, was the most cited. The article demonstrated that FUNDC1, a complete mitochondrial outer membrane protein, is a hypoxiainduced mitophagy receptor. The protein interacts with LC3 through its typical LC3 binding motif Y (18) XXL (21). The research further reveals that hypoxia induces the dephosphorylation of FDDC1 and enhances its interaction with LC3, thus achieving selective mitochondrial autophagy. These findings could provide helpful tips for mitochondrial quality control in mammalian cells.

\section{Comparison of Co-Occurring Keywords in the Field of Mitophagy}

To determine the direction of research and keyword changes in the field of mitophagy, we used the VOSViewer software to analyze the distribution of co-occurring keywords. The 


\section{A The number of articles from different journals in world}
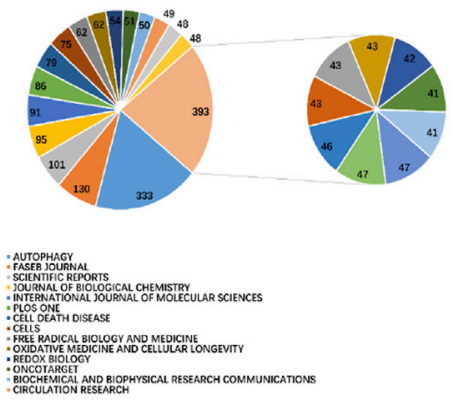

C
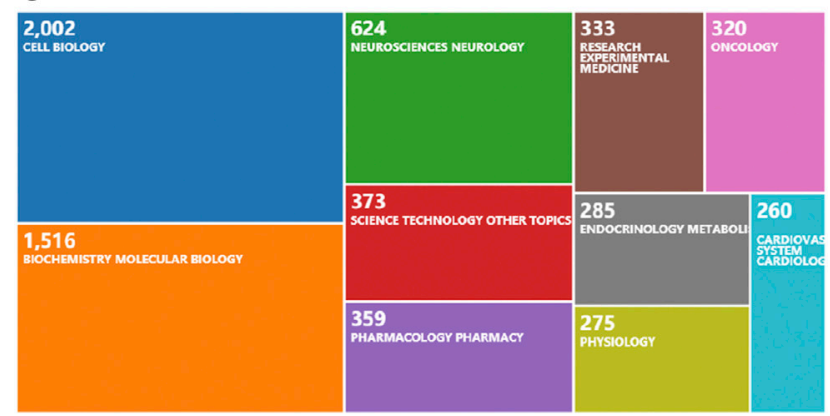

B The number of articles from different journals in China
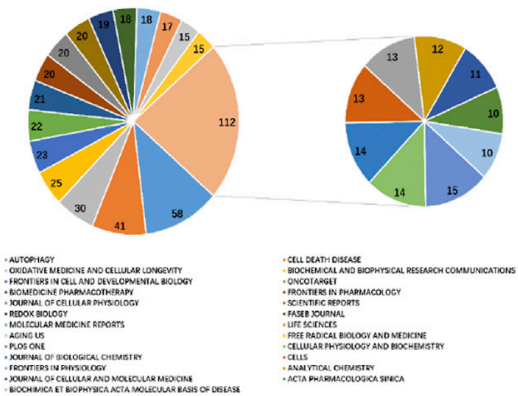

D
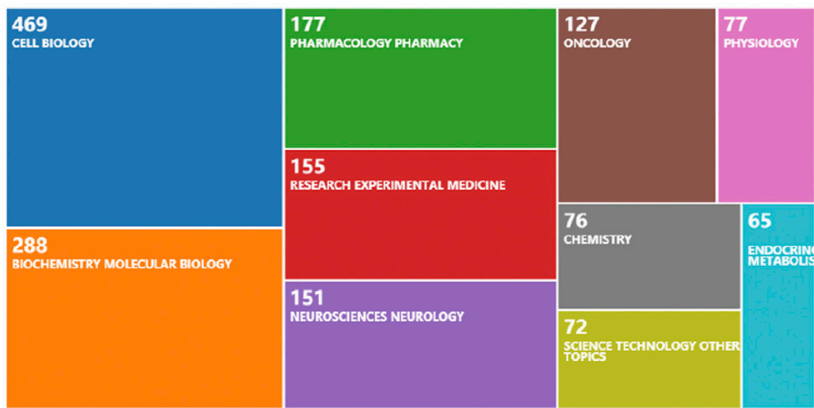

FIGURE 3 | Research direction in the field of mitophagy and the distribution of journals. (A) The number of articles from different journals across the world. (B) The number of articles from different journals in China. (C) Research direction in the field of mitophagy worldwide. (D) Research direction in the field of mitophagy in China.

TABLE 1 | The top 10 most cited articles on mitophagy research in the world.

\begin{tabular}{|c|c|c|c|c|c|}
\hline Title & Authors & $\begin{array}{l}\text { Total } \\
\text { citations }\end{array}$ & $\begin{array}{l}\text { Average } \\
\text { per } \\
\text { year }\end{array}$ & Source title & $\begin{array}{l}\text { Publication } \\
\text { date }\end{array}$ \\
\hline $\begin{array}{l}\text { A role for mitochondria in NLRP3 inflammasome } \\
\text { activation }\end{array}$ & $\begin{array}{l}\text { Zhou, Rongbin, } \\
\text { et al. }\end{array}$ & 2,477 & 225.18 & NATURE & JAN 132011 \\
\hline Mechanisms of mitophagy & $\begin{array}{l}\text { Youle, Richard } \\
\text { J., et al. }\end{array}$ & 1,680 & 152.73 & $\begin{array}{l}\text { NATURE REVIEWS MOLECULAR CELL } \\
\text { BIOLOGY }\end{array}$ & JAN 2011 \\
\hline $\begin{array}{l}\text { PINK1/Parkin-mediated mitophagy is dependent on } \\
\text { VDAC1 and p62/SQSTM1 }\end{array}$ & $\begin{array}{l}\text { Geisler, Sven, } \\
\text { et al. }\end{array}$ & 1,589 & 132.42 & NATURE CELL BIOLOGY & FEB 2010 \\
\hline $\begin{array}{l}\text { PINK1 Is Selectively Stabilized on Impaired Mitochondria } \\
\text { to Activate Parkin }\end{array}$ & $\begin{array}{l}\text { Narendra, Derek } \\
\text { P., et al. }\end{array}$ & 1,554 & 129.5 & PLOS BIOLOGY & JAN 2010 \\
\hline $\begin{array}{l}\text { Phosphorylation of ULK1 (hATG1) by AMP-Activated } \\
\text { Protein kinase Connects Energy Sensing to Mitophagy }\end{array}$ & $\begin{array}{l}\text { Egan, Daniel F., } \\
\text { et al. }\end{array}$ & 1,434 & 130.36 & SCIENCE & JAN 282011 \\
\hline Selective degradation of mitochondria by mitophagy & Kim, Insil, et al. & 1,032 & 68.8 & $\begin{array}{l}\text { ARCHIVES OF BIOCHEMISTRY AND } \\
\text { BIOPHYSICS }\end{array}$ & JUN 152007 \\
\hline $\begin{array}{l}\text { PINK1 stabilized by mitochondrial depolarization recruits } \\
\text { Parkin to damaged mitochondria and activates latent } \\
\text { Parkin for mitophagy }\end{array}$ & $\begin{array}{l}\text { Matsuda, } \\
\text { Noriyuki, et al. }\end{array}$ & 1,024 & 85.33 & JOURNAL OF CELL BIOLOGY & APR 192010 \\
\hline $\begin{array}{l}\text { PINK1-dependent recruitment of Parkin to mitochondria } \\
\text { in mitophagy }\end{array}$ & $\begin{array}{l}\text { Vives-Bauza, } \\
\text { Cristofol, et al. }\end{array}$ & 973 & 81.08 & $\begin{array}{l}\text { PROCEEDINGS OF THE NATIONAL } \\
\text { ACADEMY OF SCIENCES OF THE UNITED } \\
\text { STATES }\end{array}$ & JAN 52010 \\
\hline $\begin{array}{l}\text { The ubiquitin kinase PINK1 recruits autophagy receptors } \\
\text { to induce mitophagy }\end{array}$ & $\begin{array}{l}\text { Lazarou, } \\
\text { Michael, et al. }\end{array}$ & 936 & 133.71 & NATURE & AUG 202015 \\
\hline $\begin{array}{l}\text { Mitochondrial dynamics-fusion, fission, movement, and } \\
\text { mitophagy-in neurodegenerative diseases }\end{array}$ & $\begin{array}{l}\text { Chen, Hsiuchen, } \\
\text { et al. }\end{array}$ & 868 & 66.77 & HUMAN MOLECULAR GENETICS & OCT 152009 \\
\hline
\end{tabular}

keyword threshold was identified as 50 times, i.e., the keywords in the following results appeared in the title and abstract of the published paper at least 50 times.
Figure 4 depicts 176 identified keywords that met the threshold and were classified into 4 clusters: "mitophagy", "parkin", "oxidative stress", and "neurodegeneration" 
TABLE 2 | The top 10 most cited articles on mitophagy research in China.

\begin{tabular}{|c|c|c|c|c|c|}
\hline Title & Authors & $\begin{array}{l}\text { Total } \\
\text { citations }\end{array}$ & $\begin{array}{l}\text { Average } \\
\text { per } \\
\text { year }\end{array}$ & Source title & $\begin{array}{l}\text { Publication } \\
\text { date }\end{array}$ \\
\hline $\begin{array}{l}\text { Mitochondrial outer-membrane protein FUNDC1 mediates } \\
\text { hypoxia-induced mitophagy in mammalian cells }\end{array}$ & Liu, Lei, et al. & 636 & 63.6 & NATURE CELL BIOLOGY & FEB 2012 \\
\hline $\begin{array}{l}\text { Hepatic Autophagy Is Suppressed in the Presence of Insulin } \\
\text { Resistance and Hyperinsulinemia INHIBITION OF FoxO1- } \\
\text { DEPENDENT EXPRESSION OF KEY AUTOPHAGY GENES } \\
\text { BY INSULIN }\end{array}$ & $\begin{array}{l}\text { Liu, Hui-Yu, } \\
\text { et al. }\end{array}$ & 247 & 19 & $\begin{array}{l}\text { JOURNAL OF BIOLOGICAL } \\
\text { CHEMISTRY }\end{array}$ & NOV 62009 \\
\hline $\begin{array}{l}\text { Cerebral ischemia-reperfusion-induced autophagy protects } \\
\text { against neuronal injury by mitochondrial clearance }\end{array}$ & $\begin{array}{l}\text { Zhang, } \\
\text { Xiangnan, et al. }\end{array}$ & 245 & 27.22 & AUTOPHAGY & SEP 12013 \\
\hline $\begin{array}{l}\text { ROS and Autophagy: Interactions and Molecular Regulatory } \\
\text { Mechanisms }\end{array}$ & Li, Lulu, et al. & 224 & 32 & $\begin{array}{l}\text { CELLULAR AND MOLECULAR } \\
\text { NEUROBIOLOGY }\end{array}$ & JUL 2015 \\
\hline $\begin{array}{l}\text { ULK1 translocates to mitochondria and phosphorylates } \\
\text { FUNDC1 to regulate mitophagy }\end{array}$ & $\begin{array}{l}\text { Wu, Wenxian, } \\
\text { et al. }\end{array}$ & 217 & 27.13 & EMBO REPORTS & MAY 2014 \\
\hline Mitochondrial Regulation in Pluripotent Stem Cells & Xu, Xiuling, et al. & 215 & 23.89 & CELL METABOLISM & SEP 32013 \\
\hline $\begin{array}{l}\text { A Regulatory Signaling Loop Comprising the PGAM5 } \\
\text { phosphatase and CK2 Controls Receptor-Mediated } \\
\text { Mitophagy }\end{array}$ & $\begin{array}{l}\text { Chen, Guo, } \\
\text { et al. }\end{array}$ & 208 & 26 & MOLECULAR CELL & MAY 82014 \\
\hline $\begin{array}{l}\text { Mitochondrial dysfunction in the pathophysiology of renal } \\
\text { diseases }\end{array}$ & $\begin{array}{l}\text { Che, Ruochen, } \\
\text { et al. }\end{array}$ & 193 & 24.13 & $\begin{array}{l}\text { AMERICAN JOURNAL OF } \\
\text { PHYSIOLOGY-RENAL PHYSIOLOGY }\end{array}$ & FEB 2014 \\
\hline $\begin{array}{l}\text { miR-375 Inhibits Autophagy and Reduces Viability of } \\
\text { Hepatocellular Carcinoma Cells Under Hypoxic Conditions }\end{array}$ & $\begin{array}{l}\text { Chang, Ying, } \\
\text { et al. }\end{array}$ & 193 & 19.3 & GASTROENTEROLOGY & JUL 2012 \\
\hline $\begin{array}{l}\text { DUSP1 alleviates cardiac ischemia/reperfusion injury by } \\
\text { suppressing the Mff-required mitochondrial fission and } \\
\text { Bnip3-related mitophagy via the JNK pathways }\end{array}$ & $\begin{array}{l}\text { Jin, Qinhua, } \\
\text { et al. }\end{array}$ & 186 & 46.5 & REDOX BIOLOGY & APR 2018 \\
\hline
\end{tabular}

(Figure 4A). Figure 4B shows 37 identified keywords uncovered by the keyword co-occurrence analysis of papers in China categorized into 4 clusters: "mitophagy", "parkin", "oxidative stress", and "Mitochondrial dynamics".

Comparing the 16 years that spanned research on this field in two phases, we found that the keywords also changed dynamically over time. In reality, most of the papers on mitophagy were published in the last 5 years. In general, the burgeoning status of the research on mitophagy has seen an increase in the co-occurrence of keywords. Compared to earlier mitophagy research, investigations in the last 5 years (2016-2020) were centered on "nlrp3 inflammasome", "skeletal-muscle", and "heart failure" (Figure 4C). Early-stage mitophagy research (2005-2015) had relatively fewer research hotspots (Figure 4D).

\section{DISCUSSION}

In this bibliometric analysis, we systematically retrieved and analyzed papers published in the field of mitophagy in the past 16 years and compared the research progress and gap in this field between Chinese and foreign research institutions. This exercise provides an understanding and the ability to track development trends and research hotspots.

In the past 16 years, research on mitophagy has seen continuous and rapid development worldwide. The growth of science cannot be achieved without strong economic strength. Therefore, it does not take a genius to see that the United States has the upper hand in this field. Its institutions have published the most papers and have the highest citations and $\mathrm{H}$ indexes. The imbalance of global economic power has resulted in disproportionate scientific research and progress. Developed countries are leading the global trend in the research on mitophagy. However, China's rapidly rising economic power has now seen great improvements in this field. Still, the comparison between papers published by institutions in other major countries and Chinese establishments suggests that the quality of papers published by the latter must be improved. Nevertheless, collaborations between China and the rest of the world are becoming a frequent occurrence, and many significant findings have been published in the most important journals in the field of autophagy, which contributes to the improvement in research on mitophagy in China. With growing attention and official support, progressively more investigations have been funded in this field in China, also guaranteeing China's continuous and steady progress. From the first papers published in 2005 to 1,156 papers published in 2020, remarkable achievements have been made in the field of mitophagy worldwide, providing new perspectives for the prevention and treatment of many major diseases.

Based on these studies and results, keywords on mitophagy have also changed. For example, "Exendin-4", a glucagon-like peptide-1 (GLP-1) receptor agonist, is a new keyword in this field. Meanwhile, it is also currently considered to be an effective treatment for type 2 diabetes mellitus (Schonhoff and Harms, 2018). Many studies have shown that this GLP-1 receptor agonist has neuroprotective effects in animal models of Parkinson's disease (Hölscher, 2018). Exendin-4 has also shown favorable protective effects in patients with Parkinson's disease in a phase III clinical trial (Vijiaratnam et al., 2021). Moreover, Exendin-4 can improve motor disorders in Parkinson's mice and protect dopaminergic neurons in the substantia nigra and striatum by 


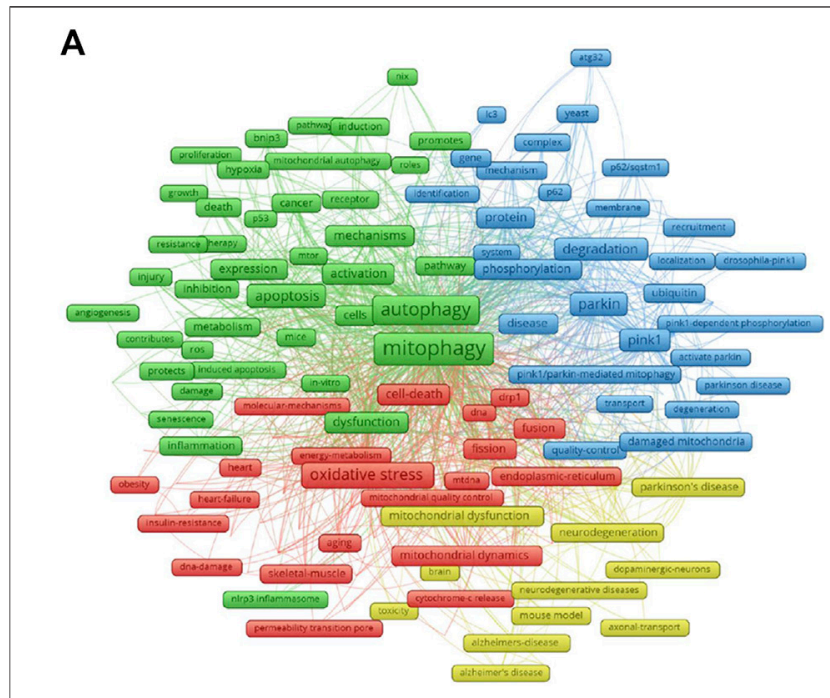

B
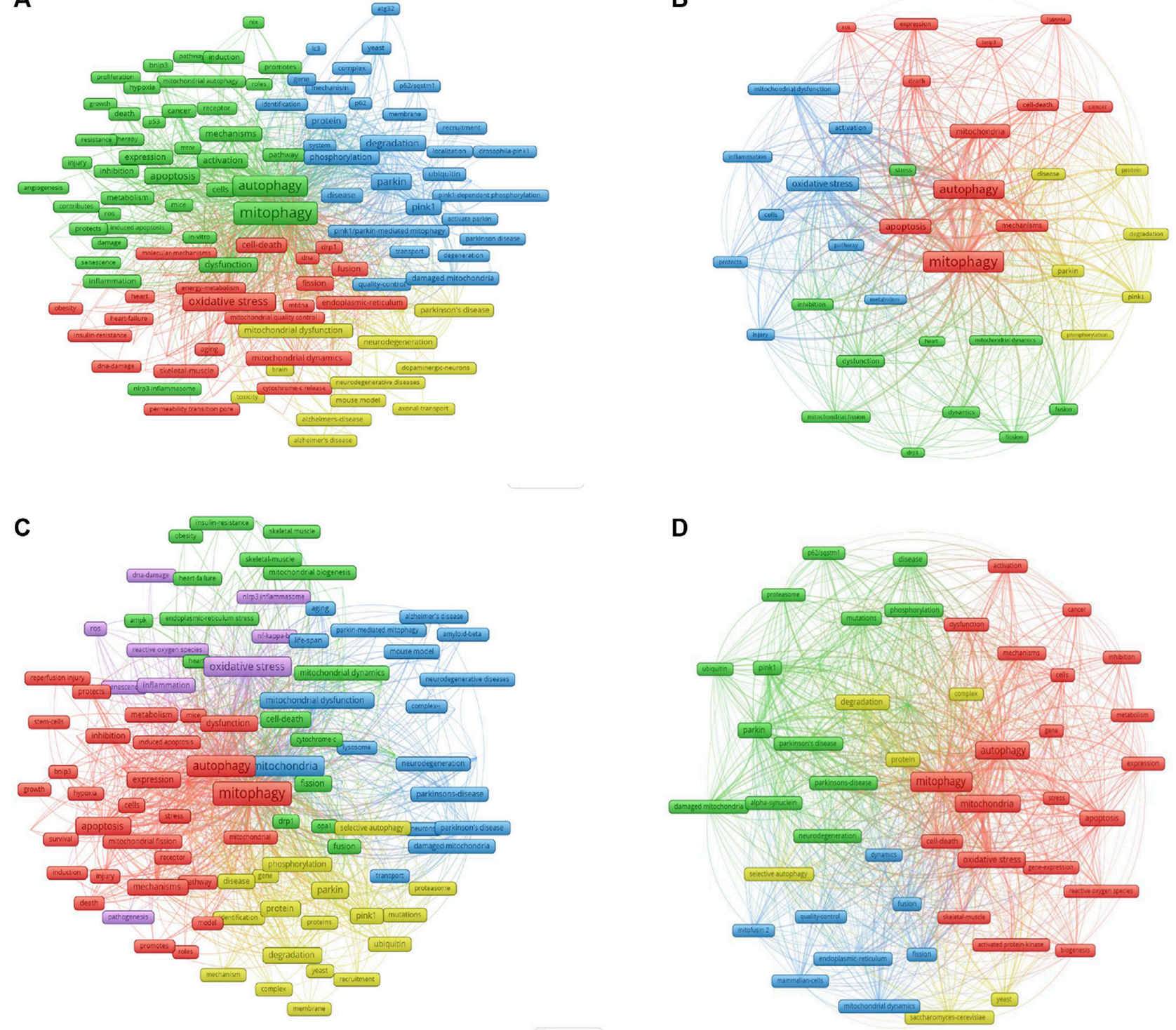

\& vosviewer

FIGURE 4 | Comparison of co-occurring keywords in the field of mitophagy. (A) Mapping of keywords in the field of mitophagy worldwide from 2005 to 2020 . (B) Mapping of keywords in the field of mitophagy in China from 2005 to 2020. (C) Mapping of keywords in the field of mitophagy from 2016 to 2020 . (D) Mapping of keywords in the field of mitophagy from 2005 to 2015.

reducing a-Syn accumulation and inhibiting mitophagy pathways. In animal models of nonalcoholic fatty liver disease, we found that GLP-1 receptor agonists protected the livers of mice with nonalcoholic fatty liver disease by enhancing the liver autophagy/mitophagy pathway, reducing oxidative stress damage, and inhibiting NLRP3 inflammasomes (Yu et al., 2019).

Recently, SIRT3 has received more and more mention in the field of mitophagy. SIRT3 is a class III histone deacetylase
(HDAC) and a part of the sirtuin gene family predominantly located in mitochondria. It is highly expressed in the brain and could eliminate reactive oxygen species, inhibit cell apoptosis, and prevent the formation of cancer cells (Yang et al., 2019). SIRT3 reportedly has a profound effect on nuclear gene expression, cancer, cardiovascular disease, neuroprotection, aging, and metabolic control (Yu et al., 2017; Lu et al., 2019; Zhou et al., 2019). According to $\mathrm{Yu}$ et al., SIRT3 deficiency aggravates 
diabetic cardiac dysfunction. Their results also suggested that inhibiting mitophagy down-regulation mediated by SIRT3FoxO3A-Parkin signaling could play a role in the pathogenesis of diabetic cardiomyopathy (Yu et al., 2017). We have also revealed in the past that SIRT3-mediated mitophagy reduces the apoptosis of tumor cells in hypoxia environments, which provides new ideas for tumor prevention and treatment (Zhou et al., 2019). Furthermore, for non-alcoholic fatty liver disease, SIRT3 overexpression can protect liver cells by promoting BNIP3-mediated-mitophagy and reducing mitochondrial apoptosis, thus effectively treating non-alcoholic cirrhosis ( $\mathrm{Li}$ et al., 2018).

Along with using the keyword co-occurrence analysis, many researchers have predominantly used "nlrp3 inflammasome" for several years. NLRP3 inflammasome is an essential component of innate immunity that can be activated by multiple types of pathogens or danger signals, such as potassium ions, mtROS, and lysosomes. NLRP3 inflammasome is key to a variety of disease processes: from the first identified familial recurrent auto-inflammatory responses to type 2 diabetes, Alzheimer's disease, and atherosclerosis (Yu et al., 2017; Li et al., 2018; Liu et al., 2019; Lu et al., 2019). Several papers have also associated the SIRT3-mitophagy-NLRP3 axis with these diseases, and it is becoming a potential therapeutic target. Per Traba et al.'s clinical investigation, fasting ameliorates the NLRP3 inflammasome activation, in part through SIRT3-mediated mitochondrial homeostatic control. The authors also suggested that deacetylase-dependent inflammasome decay could be targeted in human diseases, reflecting the prominence of the research on mitophagy currently (Traba et al., 2015).

Over the last few years, the remarkable progress in the field of mitophagy in China has correlated massively with the country's economic strength and huge investment in scientific research. Research on mitophagy in China now involves a progressively increasing number of diseases, providing a new perspective and a fresh thinking approach in the battle for the prevention and treatment of related diseases.

Still, the gap between China and world leaders in the field cannot be ignored. While the total number of papers published by Chinese institutions has increased significantly in recent years, the frequency of citation and $\mathrm{H}$-indexes remain low compared to those of its counterparts. In our bibliometric analysis, the gap between China and major countries in the world is also reflected in the journals in which the papers are published. Many papers in China are published in highly recognized journals, such as AUTOPHAGY. But these journals are mostly foreign journals. Therefore, if China wants to expand the influence of its research, it must also seek to establish recognized international journals in the field of mitophagy that can attract reputable research submissions and encourage academic cooperation.

Furthermore, we identified a substantial amount of publications on traditional Chinese medicine (TCM), which provides a unique opportunity for the development of mitophagy and traditional Chinese medicine research in tandem in China. TCM has a long history in China, and many TCM compounds are considered promising enough to be developed into approaches to treat cardiovascular and neurological diseases. However, due to the unclear molecular target, incorrect dosage, and high toxicity, the development of TCM has stagnated. Perhaps Chinese researchers combining TCM pharmacology with mitophagy could provide a remarkable opportunity to uncover promising breakthroughs in the prevention and treatment of some major diseases.

Admittedly, there are some limitations to our analysis. Firstly, bibliometric analyses cannot reflect the quality of published papers fully. And the number of citations and $\mathrm{H}$-indexes only reflect the quality of a paper from a certain aspect. Secondly, the WOS database is known for its considerably slow action in adding already existing data to its database; hence, we may not have scrutinized all already published papers not yet included in the WOS database. Thirdly, only English papers were assessed, and no retrieval analysis was conducted for papers published in other languages. Additionally, we only used the VOSViewer software for keyword co-occurrence analysis, even though many bibliometric analysis tools exist. Interpretations of our findings must, therefore, be carried out with caution.

\section{CONCLUSION}

The US and its research institutions have published the most papers, while the most common research category is cell biology. And AUTOPHAGY is the most popular journal for publishing most studies on mitophagy. We have also listed the most cited literature worldwide and in China, with the latter emerging as an increasingly powerful force in research on mitophagy, as state funding has increased, albeit still with a gap to close to major countries. This analysis presents a comprehensive representation of the state of research on mitophagy reflecting the development and trend in the field, which should help guide researchers in selecting topics.

\section{AUTHOR CONTRIBUTIONS}

Conceptualization, JY and XL software, JC; validation, JY and YJ; investigation, JC and XL; resources, XL; data curation, JY and XL; writing-original draft preparation, JC and XL; writing-review and editing, ZX; supervision, JY; funding acquisition, ZX All authors have read and agreed to the published version of the manuscript. All authors reviewed and approved the final version of the manuscript.

\section{FUNDING}

This study was supported by the National Natural Science Foundation of China (Grant Nos. 81671891 and 82101253) and the Fundamental Research Funds for the Central Universities (2042021kf0104).

\section{ACKNOWLEDGMENTS}

Thanks for the technical support from the central lab of Renmin Hospital of Wuhan University. 


\section{REFERENCES}

Bernard, C., Pommier, R., Vilgrain, V., and Ronot, M. (2019). Gender gap in Articles Published in European Radiology and CardioVascular and Interventional Radiology: Evolution between 2002 and 2016. Eur. Radiol. 30, 1011-1019. doi:10.1007/s00330-019-06390-7

Cai, Q., Zakaria, H. M., and Sheng, Z.-H. (2012). Long Time-Lapse Imaging Reveals Unique Features of PARK2/Parkin-Mediated Mitophagy in Mature Cortical Neurons. Autophagy 8 (6), 976-978. doi:10.4161/auto.20218

Chang, L., Su, Y., Zhu, R., and Duan, Z. (2019). Mapping International Collaboration in Tuberculosis Research from 1998 to 2017. Medicine (Baltimore) 98 (37), e17027. doi:10.1097/md.0000000000017027

D’Acunzo, P., Strappazzon, F., Caruana, I., Meneghetti, G., Di Rita, A., Simula, L., et al. (2019). Reversible Induction of Mitophagy by an Optogenetic Bimodular System. Nat. Commun. 10 (1), 1533. doi:10.1038/s41467-019-09487-1

Dagda, R. K., Cherra, S. J., 3rd, Kulich, S. M., Tandon, A., Park, D., and Chu, C. T. (2009). Loss of PINK1 Function Promotes Mitophagy through Effects on Oxidative Stress and Mitochondrial Fission. J. Biol. Chem. 284 (20), 13843-13855. doi:10.1074/jbc.m808515200

Doxaki, C., and Palikaras, K. (2020). Neuronal Mitophagy: Friend or Foe? Front Cel Dev Biol 8, 611938. doi:10.3389/fcell.2020.611938

Garcovich, D., and Adobes Martin, M. (2019). Measuring the Social Impact of Research in Paediatric Dentistry: An Altmetric Study. Int. J. Paediatr. Dent. 30, 66-74. doi:10.1111/ipd.12575

Geisler, S., Holmström, K. M., Skujat, D., Fiesel, F. C., Rothfuss, O. C., Kahle, P. J., et al. (2010). PINK1/Parkin-mediated Mitophagy Is Dependent on VDAC1 and p62/SQSTM1. Nat. Cel Biol 12 (2), 119-131. doi:10.1038/ncb2012

Hölscher, C. (2018). Novel Dual GLP-1/GIP Receptor Agonists Show Neuroprotective Effects in Alzheimer's and Parkinson's Disease Models. Neuropharmacol. 136 (Pt B), 251-259. doi:10.1016/j.neuropharm.2018.01.040

Iwata, K. (2019). Mitochondrial Involvement in Mental Disorders; Energy Metabolism, Genetic, and Environmental Factors. Methods Mol. Biol. 1916, 41-48. doi:10.1007/978-1-4939-8994-2_2

Lei, F., Ye, J., Wang, J., and Xia, Z. (2019). A Bibliometric Analysis of Publications on Oxycodone from 1998 to 2017. Biomed. Res. Int. 2019, 9096201. doi:10.1155/ 2019/9096201

Li, R., Xin, T., Li, D., Wang, C., Zhu, H., and Zhou, H. (2018). Therapeutic Effect of Sirtuin 3 on Ameliorating Nonalcoholic Fatty Liver Disease: The Role of the ERK-CREB Pathway and Bnip3-Mediated Mitophagy. Redox Biol. 18, 229-243. doi:10.1016/j.redox.2018.07.011

Liu, L., Feng, D., Chen, G., Chen, M., Zheng, Q., Song, P., et al. (2012). Mitochondrial Outer-Membrane Protein FUNDC1 Mediates HypoxiaInduced Mitophagy in Mammalian Cells. Nat. Cel Biol 14 (2), 177-185. doi:10.1038/ncb2422

Liu, Y., Cheng, A., Li, Y.-J., Yang, Y., Kishimoto, Y., Zhang, S., et al. (2019). SIRT3 Mediates Hippocampal Synaptic Adaptations to Intermittent Fasting and Ameliorates Deficits in APP Mutant Mice. Nat. Commun. 10, 1886. doi:10.1038/s41467-019-09897-1

Lu, H., Sun, L., Chen, W., Zhou, Y., Liu, K., Chen, J., et al. (2019). Sirtuin 3 Therapy Attenuates Aging Expression, Oxidative Stress Parameters and Neointimal Hyperplasia Formation in Vein Grafts. Ann. Vasc. Surg. 64, 303-317. doi:10.1016/j.avsg.2019.05.044

Nakao, S., Yamamoto, T., Kimura, S., Mino, T., and Iwamoto, T. (2019). Brain white Matter Lesions and Postoperative Cognitive Dysfunction: a Review. J. Anesth. 33 (2), 336-340. doi:10.1007/s00540-019-02613-9

Pamenter, M. E., Lau, G. Y., and Richards, J. G. (2018). Effects of Cold on Murine Brain Mitochondrial Function. PLoS One 13 (12), e0208453. doi:10.1371/ journal.pone. 0208453

Salimi, A., Nikoosiar Jahromi, M., and Pourahmad, J. (2019). Maternal Exposure Causes Mitochondrial Dysfunction in Brain, Liver, and Heart of Mouse Fetus: An Explanation for Perfluorooctanoic Acid Induced Abortion and Developmental Toxicity. Environ. Toxicol. 34 (7), 878-885. doi:10.1002/tox.22760

Schonhoff, A. M., and Harms, A. S. (2018). Glial GLP1R: A Novel Neuroprotector? Mov Disord. 33 (12), 1877. doi:10.1002/mds.27547
Traba, J., Kwarteng-Siaw, M., Okoli, T. C., Li, J., Huffstutler, R. D., Bray, A., et al. (2015). Fasting and Refeeding Differentially Regulate NLRP3 Inflammasome Activation in Human Subjects. J. Clin. Invest. 125, 4592-4600. doi:10.1172/ jci83260

Vijiaratnam, N., Girges, C., Auld, G., Chau, M., Maclagan, K., King, A., et al. (2021). Exenatide once Weekly over 2 Years as a Potential Disease-Modifying Treatment for Parkinson's Disease: Protocol for a Multicentre, Randomised, Double Blind, Parallel Group, Placebo Controlled, Phase 3 Trial: The 'Exenatide-PD3' Study. BMJ Open 11, e047993. doi:10.1136/bmjopen-2020047993

Wang, K., Xing, D., Dong, S., and Lin, J. (2019). The Global State of Research in Nonsurgical Treatment of Knee Osteoarthritis: a Bibliometric and Visualized Study. BMC Musculoskelet. Disord. 20, 407. doi:10.1186/s12891-019-2804-9

Wang, X. L., Feng, S. T., Wang, Y. T., Yuan, Y. H., Li, Z. P., Chen, N. H., et al. (2021). Mitophagy, a Form of Selective Autophagy, Plays an Essential Role in Mitochondrial Dynamics of Parkinson's Disease. Cell Mol Neurobiol. doi:10.1007/s10571-021-01039-w

Yang, Y., Li, N., Chen, T., Zhang, C., Li, J., Liu, L., et al. (2019). Sirt3 Promotes Sensitivity to Sunitinib-Induced Cardiotoxicity via Inhibition of GTSP1/JNK/ autophagy Pathway In Vivo and In Vitro. Arch. Toxicol. 93, 3249-3260. doi:10.1007/s00204-019-02573-9

Ye, J., Ding, H., Ren, J., and Xia, Z. (2018). The Publication Trend of Neuropathic Pain in the World and China: a 20-years Bibliometric Analysis. J. Headache Pain 19 (1), 110. doi:10.1186/s10194-018-0941-4

Ye, J. S., Chen, L., Lu, Y. Y., Lei, S. Q., Peng, M., and Xia, Z. Y. (2019). HonokiolMediated Mitophagy Ameliorates Postoperative Cognitive Impairment Induced by Surgery/Sevoflurane via Inhibiting the Activation of NLRP3 Inflammasome in the Hippocampus. Oxid Med. Cel Longev 2019, 8639618. doi:10.1155/2019/8639618

Yu, W., Gao, B., Li, N., Wang, J., Qiu, C., Zhang, G., et al. (2017). Sirt3 Deficiency Exacerbates Diabetic Cardiac Dysfunction: Role of Foxo3A-Parkin-Mediated Mitophagy. Biochim. Biophys. Acta (Bba) - Mol. Basis Dis. 1863 (8), 1973-1983. doi:10.1016/j.bbadis.2016.10.021

Yu, X., Hao, M., Liu, Y., Ma, X., Lin, W., Xu, Q., et al. (2019). Liraglutide Ameliorates Non-alcoholic Steatohepatitis by Inhibiting NLRP3 Inflammasome and Pyroptosis Activation via Mitophagy. Eur. J. Pharmacol. 864, 172715. doi:10.1016/j.ejphar.2019.172715

Zhao, F., Du, F., Shi, D., Zhou, W., Jiang, Y., and Ma, L. (2019). Mapping Research Trends of Retinal Vein Occlusion from 2009 to 2018: a Bibliometric Analysis. PeerJ 7, e7603. doi:10.7717/peerj.7603

Zhou, J., Shi, M., Li, M., Cheng, L., Yang, J., and Huang, X. (2019). RETRACTED ARTICLE: Sirtuin 3 Inhibition Induces Mitochondrial Stress in Tongue Cancer by Targeting Mitochondrial Fission and the JNK-Fis1 Biological axis. Cell Stress and Chaperones 24 (2), 369-383. doi:10.1007/s12192-019-00970-8

Zilocchi, M., Fasano, M., and Alberio, T. (2019). Mitochondrial Proteins in the Development of Parkinson's Disease. Adv. Exp. Med. Biol. 1158, 17-44. doi:10.1007/978-981-13-8367-0_2

Conflict of Interest: The authors declare that the research was conducted in the absence of any commercial or financial relationships that could be construed as a potential conflict of interest.

Publisher's Note: All claims expressed in this article are solely those of the authors and do not necessarily represent those of their affiliated organizations, or those of the publisher, the editors and the reviewers. Any product that may be evaluated in this article, or claim that may be made by its manufacturer, is not guaranteed or endorsed by the publisher.

Copyright (C) 2021 Chen, Li, Jia, Xia and Ye. This is an open-access article distributed under the terms of the Creative Commons Attribution License (CC BY). The use, distribution or reproduction in other forums is permitted, provided the original author(s) and the copyright owner(s) are credited and that the original publication in this journal is cited, in accordance with accepted academic practice. No use, distribution or reproduction is permitted which does not comply with these terms. 